\title{
A Novel Fuzzy Synthetic Discrimination System for Teaching Ability of Physical Education Majors Based on Analytic Hierarchy Process
}

\author{
Yimeng Fan ${ }^{1}$ \\ Hebei Agricultural \\ University \\ Lili Zhao ${ }^{4}$ \\ Hebei Agricultural \\ University
}

\author{
Jiangwei Chen ${ }^{2}$ \\ Hebei Agricultural \\ University
}

Yanping Zhang ${ }^{5}$

Hebei Agricultural University

\author{
Songfeng $\mathrm{Li}^{3}$ \\ Hebei Agricultural \\ University \\ Chunming $\mathrm{Liu}^{6}$ \\ Hebei Agricultural \\ University
}

\begin{abstract}
Considering the importance of teaching ability among physical education (PE) majors, this paper relies on analytic hierarchy process (AHP) and multi-level fuzzy synthetic discrimination (FSD) to establish an evaluation index system for the teaching ability of PE majors. The established system involves 6 primary indices, 21 secondary indices and 66 tertiary indices. The weight of each index was determined through both qualitative and quantitative analyses. This research provides theoretical basis for PE teaching.
\end{abstract}

\section{Keywords}

Physical Education (PE) • Teaching Ability • Analytic Hierarchy Process (AHP) • Fuzzy Synthetic Discrimination (FSD)

\footnotetext{
*Teaching management special item (2016GL07): Construction of teaching information feedback system for college students ${ }^{1}$ Department of Sports Works, Hebei Agricultural University, Baoding 071000, China. Email: fanyimeng@126.com ${ }^{2}$ Academic Affairs Office, Hebei Agricultural University, Baoding 071000, China. Email: faeriewei@ hotmail.com ${ }^{3}$ Department of Sports Works, Hebei Agricultural University, Baoding 071000, China. Email: 326911945@qq.com ${ }^{4}$ Department of Sports Works, Hebei Agricultural University, Baoding 071000, China. Email: 31883920@qq.com ${ }_{5}^{5}$ Department of Sports Works, Hebei Agricultural University, Baoding 071000, China. Email: zyp651008@ 126.com ${ }^{6}$ Correspondence to: Chunming Liu (BA), Department of Sports Works, Hebei Agricultural University, Baoding 071000, China. Email: tyliu7526143@163.com
} 
Fan, Chen, Li, Zhao, Zhang, Lin / A Novel Fuzzy Synthetic Discrimination System for Teaching Ability of...

Physical education (PE) is a multi-functional system that lays the basis for all-round education. Therefore, it is important to cultivate PE majors into qualified teachers. To this end, PE majors must acquire the basic knowledge and skills of PE and develop a good teaching ability based on these knowledge and skills. The teaching ability determines whether a PE teacher can adapt to the development of the discipline. Despite the importance of teaching ability, there are still many problems in the current evaluation methods for PE majors.

In essence, any evaluation is a valuation of the object (Aghaei, 2013). Modern theories on education evaluation often attach equal importance to the teaching results and the teaching process. However, the current evaluation methods for PE majors emphasises on physical quality and skills over teaching ability, failing to objectively reflect the efforts and progress of the objects. This calls for an evaluation system that can scientifically and accurately measure the teaching ability of PE majors.

Since teaching ability involves multiple fuzzy factors, qualitative evaluation often leads to subjective and one-sided conclusions. This defect cannot be solved by accurate mathematical method, but the combination between the fuzzy synthetic discrimination (FSD) based on analytic hierarchy process (AHP). Inspired by fuzzy membership theory, the integrated method can quantify the qualitative indices and combine the merits of qualitative and quantitative evaluations. Hence, this paper adopts an AHP-based FSD to establish an evaluation index system for the teaching ability of PE majors, aiming to provide theoretical basis for PE teaching.

\section{Literature Review}

The teaching ability of PE teachers is fundamental to the success of PE. Over the years, much research has been done on the evaluation of teaching ability. For instance, Lu (2007) explored the teaching ability of PE majors based on the frequency of teaching practice, and concluded that the teaching ability covers 11 aspects, such as demonstration ability, explanation ability, error correction ability and organizational ability (Lu, 2007). Hui (2008) elaborated the contents of teaching ability in such dimensions as knowledge structure, basic skills, teaching mood, temperature and character, reputation, etc. (Hui, 2008). Huang, Song \& Guo (2007) held that the teaching ability of PE majors involves document preparation, demonstration, organization, error discovery and correction, and contingency handling. Sun (2004) divided the teaching ability of PE majors into the ability to design, organize, evaluate and analyse PE teaching activities (Sun, 2004).

Many scholars have also probed into the training of teaching ability of PE majors. For example, Liu (2004) conducted a survey on the teaching practice of PE majors, and relied on images to analyse the factors of their teaching ability. Franco \& Bras (2010) identified the main components of teaching ability, and then determined the main contents, schedule, methods and evaluation strategies for teaching ability training. To solve the lack of teaching ability among PE majors, Zou (2014) proposed to adjust the teaching progress and implement physical fitness training in a planned way (Zou, 2014). Zhao et al., (2010) also pointed out the necessity to train the teaching ability of PE majors in a planned manner. 
To sum up, the existing studies mainly concentrate on qualitative evaluation about the nature and training methods of PE teaching ability. Besides, there are many theoretical researches and few empirical studies. Most researches only have some logical reasoning on the basic theory of teaching ability.

\section{Methodology}

\section{Fuzzy Mathematics}

As its name suggests, fuzzy mathematics is a mathematical theory and method to tackle fuzziness (Buckley, 1993). It is an extension of exact mathematics because of its accuracy. Fuzzy mathematics methods are applicable to the judgement, evaluation, reasoning, decision-making and control based on fuzzy concepts, provided that these concepts can be described by the fuzzy set. The typical approaches of fuzzy mathematics include the FSD, fuzzy clustering analysis, fuzzy pattern recognition, fuzzy decision-making and fuzzy prediction, fuzzy control, fuzzy information processing and so on. Together, these methods constitute a fuzzy system theory (Chen, 2003). Fuzzy mathematics methods have already been widely adopted for medicine, meteorology, psychology, economic management, education and sports.

\section{AHP}

Analytic hierarchy process (AHP) is a decision-making method that qualifies and quantifies the decisionmaking elements like goals, criteria and alternatives (Saaty, 2008). Taking a complex multi-objective decisionmaking problem as a system, the AHP decomposes the objective into multiple goals, and further divide the problem into several index layers (or criteria and constraints). Then, the single ranking (weights) and the general ranking of qualitative indices are calculated by fuzzy quantitative method, and used to optimize the decisionmaking based on multiple alternatives.

The AHP is particularly suitable for a system whose evaluation indices are layered and interlaced and whose objective value is difficult to quantify. First, the decision-making problem can be divided into different layers by the general goal, the target of each layer, the evaluation criterion and the specific alternative. Then, the priority weight of each upper level element corresponding to an element can be obtained by determining the eigenvector of matrix. Finally, the final weights of all alternatives to the general goal can be merged by the weighted sum method.

An important feature of the AHP is the importance degree ratio between two alternatives. For a certain criterion, all alternatives are contrasted with each other and evaluated by importance. The resulting matrix is called the judgment matrix. For the judgement matrix, it is necessary to obtain the eigenvector corresponding to the maximum eigenvalue and normalize it as weight. In this way, the weight vector of the judgement matrix can be obtained, making it possible to extract useful information from the matrix and lay a scientific basis for decision-making.

\section{Multi-level FSD}

The FSD is a comprehensive evaluation of some vague objects (Jin, Wei, \& Jing, 2004). This method 
combines the AHP and fuzzy membership theory to reasonably quantify the qualitative indices. An AHP-based FSD model consists of two complementary parts, namely, the AHP and the FSD. The model can be implemented in three steps: division of factor layer and determination of the criterion set, construction of membership function and factor weight set by the AHP, and finally the comprehensive evaluation.

The basic idea of comprehensive evaluation lies in the judgement of all factors related to the objects by linear and maximum subordinate principle. Let $A_{i}$ be evaluation objects, $\mathrm{U}=\left\{u_{1}, u_{2}, \ldots, u_{m}\right\}$ be factors set with $\mathrm{m}$ being the number of factors related to $A_{i}$, and $\mathrm{V}=\left\{v_{1}, v_{2}, \ldots, v_{n}\right\}$ be the evaluation set with $\mathrm{n}$ being the number of possible evaluations. To determine a relation $R \in \mu_{m \times n}, \mathrm{~F}$ mappings are required, forming an evaluation matrix. Let $\mathrm{W}=\left\{w_{1}, w_{2}, \ldots, w_{m}\right\}$ be the weight distribution of various factors. Then, comprehensive evaluation set $\mathrm{B}=\left\{b_{1}, b_{2}, \ldots, b_{n}\right\}$ can be obtained from $W$ and $R$. According to the principle of maximum membership, the evaluation $v_{i}$ corresponding to the largest $b_{i}$ in $\mathrm{B}$ should be selected as the result of comprehensive evaluation. The above process can be expressed as follows:

$$
\begin{gathered}
\mathrm{R}=\left[\begin{array}{cccc}
r_{11} & r_{12} & \ldots & r_{1 n} \\
r_{21} & r_{22} & \ldots & r_{2 n} \\
\ldots & \ldots & \ldots & \ldots \\
r_{m 1} & r_{m 2} & \ldots & r_{m n}
\end{array}\right] \\
\mathrm{W} \circ \mathrm{R}=\mathrm{B}=\left\{b_{1}, b_{2}, \ldots, b_{n}\right\}
\end{gathered}
$$

\section{Evaluation Index System}

Through literature review and expert consultation, several evaluation indices were determined for the teaching ability of PE majors. Each index was rated by a 5-point scale as follows: 1. "extremely important", 2. "very important", 3. "important", 4. "general" and 5. "unimportant". Then, the score of each index was counted and evaluated by the experts again. The index levels and contents were adjusted to facilitate weight calculation and rationalize the index classification. Considering the strong fuzziness of some evaluation indices of PE teaching ability, the final index system (Figure 1) was established based on expert opinions and the knowledge system of the PE discipline. The final system contains three levels of indices, including 6 primary indices, 21 secondary indices and 66 tertiary indices.

\section{Expressive ability}

Expressive ability, especially the clarity of oral explanation, is an important means for the teacher to impart knowledge and skills. Essential to the teaching effect, this ability is a key measure of the teaching ability of PE majors.

\section{Demonstration ability}

Demonstration is the most fundamental teaching method of PE. Correct and standardized demonstration can provide students with the most intuitive visual stimulation, arousing their interests in learning. 


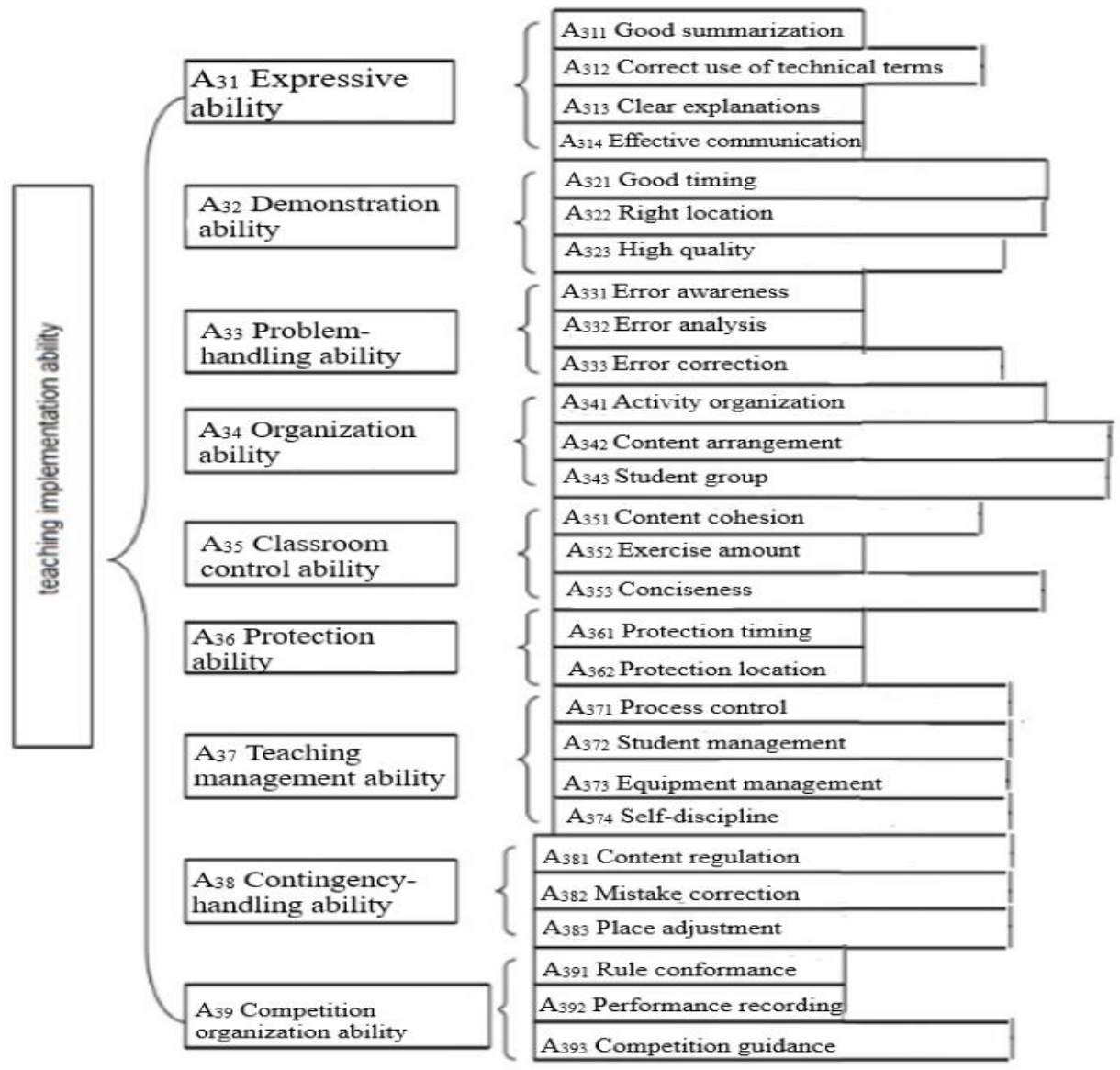

Figure 1. Evaluation index system for teaching ability of PE majors.

\section{Problem-handling ability}

The problem-handling ability mainly refers to the awareness of the wrong actions in PE teaching. In the course of teaching, the PE majors should identify and correct their mistakes, and find out the causes of these wrong actions.

\section{Organization ability}

Statistics show that the organization ability is a key factor in the performance of classroom teaching. Good PE teachers should be able to manage the complex equipment and facilities in the venues of PE classes, such as sports field or the gym.

\section{Classroom control ability}

To optimize the structure of classroom teaching, the teaching contents must be arranged according to the acceptance ability and cognitive level of the students, and special attention must be paid to the cohesion between 
old and new contents.

\section{Protection ability}

During PE classes, the teacher should provide protection to students at the right moments. The protective actions help the students overcome fear and avoid accidents. Thus, the protection ability is an important guarantee of the teaching effect.

\section{Teaching management ability}

Education management ability stands for the ability to apply management theories and methods in the course of teaching. This ability is the direct result of the continuous learning of the knowledge on management science.

\section{Contingency-handling ability}

Contingency-handling ability refers to the teacher's ability to handle unexpected incidents in class. Through proper contingency-handling, it is possible for the teacher to realize smooth teaching and good teaching effect.

\section{Competition organization ability}

Competition is a good way to arouse the enthusiasm of students. Thus, the teacher's ability to organize competitions directly bears on the teaching effect and should be regarded as an indicator of PE masters' teaching ability.

\section{Construction of FSD System}

\section{Fuzzy judgment matrix}

The fuzzy judgment matrix is generally created in three steps: calculating the weight index layers, determine the layers of AHP comparison matrix, and setting up the fuzzy judgement matrix according to the layers of the corresponding weight indices. Through pairwise comparison of the said evaluation indices, the fuzzy judgement matrix in this paper was constructed against Saaty’s 1 9 level relative importance scale (Saaty, 1994) (Table $1)$.

For $\mathrm{n}$ indices on the same level, the fuzzy judgment matrix $A=\left(a_{i j}\right)_{n n}, a_{i j}>0, a_{i i}=1$ can be obtained.

The eigenvector of the fuzzy judgment matrix can be calculated as:

$$
W_{i}=\frac{\overline{W_{l}}}{\sum_{i=1}^{n} W_{i}}
$$

Table 1

Saaty's Relative Importance Scale

\begin{tabular}{ll}
\hline Scale & Description \\
\hline 1 & The two indices are equally important. \\
3 & One index is slightly more important than the other. \\
5 & One index is significantly more important than the other. \\
7 & One index is slightly less important than the other. \\
9 & One index is significantly less important than the other. \\
$2,4,6$ and 8 & The middle value of the results of neighbouring scales. \\
\hline
\end{tabular}

Then, the maximum eigenvalue of judgment matrix can be calculated as: 


$$
\lambda_{\max }=\sum_{i=1}^{n} \frac{(A W)_{i}}{n W_{i}}
$$

The consistency index CI can be computed as:

$$
\mathrm{CI}=\frac{\lambda_{\max }-n}{n-1}
$$

The average consistency index RI of the same order matrix is shown in Table 2.

Table 2

The Average Consistency Index RI

\begin{tabular}{lcccccccccc}
\hline Order $n$ & 1 & 2 & 3 & 4 & 5 & 6 & 7 & 8 & 9 & 10 \\
\hline$R I$ & 0 & 0 & 0.56 & 0.86 & 1.17 & 1.25 & 1.33 & 1.46 & 1.49 & 1.51 \\
\hline
\end{tabular}

Note. $\mathrm{CR}=\mathrm{CI} / \mathrm{RI}$. When $\mathrm{CR}=0$, A has complete consistency. When $C R<0.1$, A has satisfactory consistency. When $C R \geq 0.1$, A has unsatisfactory consistency. Considering the evaluation indices of PE teaching ability, the fuzzy judgement matrix can be constructed according to the statistical results of the assigned scores (Table $3)$.

Table 3

Fuzzy judgment matrix of evaluation indices of $P E$ teaching ability

\begin{tabular}{lccccccccc}
\hline $\mathrm{A}$ & $A_{31}$ & $A_{32}$ & $A_{33}$ & $A_{34}$ & $A_{35}$ & $A_{36}$ & $A_{37}$ & $A_{38}$ & $A_{39}$ \\
\hline$A_{31}$ & 1 & 0.33 & 0.5 & 0.14 & 0.17 & 0.25 & 0.20 & 2 & 3 \\
$A_{32}$ & 3 & 1 & 2 & 0.2 & 0.25 & 0.5 & 0.33 & 4 & 5 \\
$A_{33}$ & 2 & 0.5 & 1 & 0.16 & 0.2 & 0.33 & 0.25 & 3 & 4 \\
$A_{34}$ & 7 & 5 & 6 & 1 & 2 & 4 & 3 & 8 & 9 \\
$A_{35}$ & 6 & 4 & 5 & 0.5 & 1 & 3 & 2 & 7 & 8 \\
$A_{36}$ & 4 & 2 & 3 & 0.25 & 0.33 & 1 & 0.5 & 5 & 6 \\
$A_{37}$ & 5 & 3 & 4 & 0.33 & 0.5 & 2 & 1 & 6 & 7 \\
$A_{38}$ & 0.5 & 0.25 & 0.33 & 0.13 & 0.14 & 0.20 & 0.17 & 1 & 2 \\
$A_{39}$ & 0.33 & 0.20 & 0.25 & 0.11 & 0.13 & 0.17 & 0.14 & 0.5 & 1 \\
\hline
\end{tabular}

Table 4 shows the product of the elements in each row of the fuzzy judgement matrix.

Table 4

Product of the Elements in Each Row of the Fuzzy Judgement Matrix

\begin{tabular}{ccccccccc}
\hline$M_{1}$ & $M_{2}$ & $M_{3}$ & $M_{4}$ & $M_{5}$ & $M_{6}$ & $M_{7}$ & $M_{8}$ & $M_{9}$ \\
\hline $1 / 840$ & 1 & $1 / 30$ & 362880 & 20160 & 30 & 840 & $1 / 20160$ & $1 / 362880$ \\
\hline
\end{tabular}

Table 5 lists the n square root of $M_{i}$.

Table 5

\begin{tabular}{|c|c|c|c|c|c|c|c|c|}
\hline$\overline{W_{1}}$ & $\overline{\overline{W_{2}}}$ & $\overline{\overline{W_{3}}}$ & $\overline{\overline{W_{4}}}$ & $\overline{\overline{W_{5}}}$ & $\overline{\overline{W_{6}}}$ & $\overline{W_{7}}$ & $\overline{\overline{W_{8}}}$ & $\overline{W_{9}}$ \\
\hline 0.471 & 1 & 0.686 & 4.146 & 3.009 & 1.461 & 2.109 & 0.333 & 0.239 \\
\hline
\end{tabular}

$n$ Square Root of $M_{i}$

According to Tables 4 and 5, the eigenvectors of the fuzzy judgment matrix were obtained as follows.

Table 6

Eigenvectors of Fuzzy Judgment Matrix

\begin{tabular}{rcccccccc}
\hline$W_{1}$ & $W_{2}$ & $W_{3}$ & $W_{4}$ & $W_{5}$ & $W_{6}$ & W & $W_{8}$ & $W_{9}$ \\
\hline 0.035 & 0.074 & 0.051 & 0.308 & 0.224 & 0.108 & 0.157 & 0.025 & 0.018 \\
\hline
\end{tabular}

\section{Construction of FSD system}

Let $\mathrm{A}=\left\{A_{1}, A_{2}, \ldots A_{j}, \ldots, A_{m}\right\}$ be the factor set, where $A_{j}$ is the $\mathrm{j}$-th factor and $\mathrm{m}$ is the total number of factors.

Let $\mathrm{P}$ be a partition of factor $\mathrm{A}$, then $U / P=\left\{A_{1}, A_{2}, A_{3}, A_{4}, A_{5}, A_{6}\right\}$, where $A_{1}=\left\{A_{11}, A_{12}\right\}, A_{2}=$ 
Fan, Chen, Li, Zhao, Zhang, Lin / A Novel Fuzzy Synthetic Discrimination System for Teaching Ability of...

$\left\{A_{21}, A_{22}, A_{23}\right\}, A_{3}=\left\{A_{31}, A_{32}, A_{33}, A_{34}, A_{35}, A_{36}, A_{37}, A_{38}, A_{39}\right\}, A_{4}=\left\{A_{41}, A_{42}, A_{43}\right\}, A_{5}=\left\{A_{51}, A_{52}, A_{53}\right\}$, and $A_{6}=\left\{A_{61}, A_{62}\right\}$.

Let $\mathrm{V}=\left\{v_{1}, v_{2}, \ldots, v_{j}, \ldots, v_{m}\right\}$ be a set of evaluations, where $v_{j}$ is the $\mathrm{j}$-th evaluation result. $\mathrm{m}$ is the total number of evaluation results. Suppose $\mathrm{V}=\{$ worst, bad, medium, good, excellent $\}=\left\{v_{1}, v_{2}, v_{3}, v_{4}, v_{5}\right\}$, and 20, 40, 60, 80 and 100 are divisions of the scoring interval. Thus, a fuzzy membership degree from $\mathrm{V}$ to $\mathrm{A}$ can be obtained (Table 7).

Table 7

Membership Degree of the Evaluation in Each Interval

\begin{tabular}{lccccc}
\hline Score & 20 & 40 & 60 & 80 & 100 \\
\hline Worst & 0.75 & 0.25 & 0 & 0 & 0 \\
Bad & 0.25 & 0.50 & 0.25 & 0 & 0 \\
Medium & 0 & 0.25 & 0.50 & 0.25 & 0 \\
Good & 0 & 0 & 0.25 & 0.50 & 0.25 \\
Excellent & 0 & 0 & 0 & 0.25 & 0.75 \\
\hline
\end{tabular}

Taking a three-level FSD model, this paper counts the membership degree of each index, and constructs the single-factor judgement matrix of the primary FSD:

$$
\mathrm{E}=W_{1} * R_{1}=(0.288,0.288,0.424)\left[\begin{array}{lllll}
r_{11} & r_{12} & r_{13} & r_{14} & r_{15} \\
r_{21} & r_{22} & r_{23} & r_{24} & r_{25} \\
r_{31} & r_{32} & r_{33} & r_{34} & r_{35}
\end{array}\right]=\left\{W_{11}, W_{12}, W_{13}, W_{14}, W_{15}\right\}
$$

The first-level FSD is only one kind of factors in the synthesis. Different types of factors should be integrated to reveal the overall impacts of various factors. Thus, we have the secondary comprehensive evaluation.

$$
R_{E}=\left(\begin{array}{l}
E_{1} \\
E_{2} \\
E_{3} \\
E_{4} \\
E_{5} \\
E_{6}
\end{array}\right)=\left(\begin{array}{l}
W_{1} * R_{1} \\
W_{2} * R_{2} \\
W_{3} * R_{3} \\
W_{4} * R_{4} \\
W_{5} * R_{5} \\
W_{6} * R_{6}
\end{array}\right)=\left(\begin{array}{lllll}
W_{11} & W_{12} & W_{13} & W_{14} & W_{15} \\
W_{21} & W_{22} & W_{23} & W_{24} & W_{25} \\
W_{31} & W_{32} & W_{33} & W_{34} & W_{35} \\
W_{41} & W_{42} & W_{43} & W_{44} & W_{45} \\
W_{51} & W_{52} & W_{53} & W_{54} & W_{55} \\
W_{61} & W_{62} & W_{63} & W_{64} & W_{65}
\end{array}\right)
$$

The tertiary FSD is shown as follows:

$$
A_{\text {com }}=\{0.045,0.116,0.398,0.072,0.156,0.217\}\left(\begin{array}{l}
R_{Z} \\
S_{Z}
\end{array}\right)
$$

where $R_{Z}$ is $R_{\text {com }}$ and $S_{Z}$ is $S_{\text {com }} . \mathrm{X}=A_{\text {com }} * C^{T}$, where C $=\left[\begin{array}{ll}20 & 406080100\end{array}\right]$ and $C^{T}$ is the transposed matrix of C. In this way, it is possible to derive the comprehensive evaluation score of PE majors' teaching ability.

\section{Conclusion}

Teaching ability is one of the most basic capabilities of PE majors. To build a scientific evaluation index system for PE teaching ability, this paper sets up an evaluation index system involving 6 primary indices, 21 secondary indices and 66 tertiary indices. The weight of each index was determined through both qualitative and quantitative analyses. Based on the AHP, the proposed FSD system can achieve high internal consistency. 


\section{References}

Aghaei, N. (2013). Relationship between organizational Empowerment and organizational citizenship behavior in physical education teachers. Zeitschrift Für Experimentelle Psychologie, 48(4), 339-364.

Buckley, J. J. (1993). The fuzzy mathematics of finance. Readings in Fuzzy Sets for Intelligent Systems, 21(3), 840-848. http://dx.doi.org/10.1016/0165-0114(87)90128-X

Chen, S. M. (2003). Analyzing fuzzy system reliability using vague set theory. International Journal of Applied Science \& Engineering, 1(1), 82-88. http://dx.doi.org/10.1109/ICSMC.1997.638243

Lu, L. F. L. (2007). Present situation investigation and countermeasure research on extracurricular specialized study of PE major students [J]. Journal of Harbin Institute of Physical Education, 5, 040.

Franco, M., \& Bras, M. D. L. (2010). Training and strategic competencies of the executive manager in schools: an exploratory case study. International Journal of Management in Education,4(4), 425-445. http://dx.doi.org/10.1504/IJMIE.2010.035609

Huang, G. L., Song, M. M., \& Guo, X. P. (2007). A synthetic study and practice of the construction of college PE curriculum system [J]. Sports Science Research, 3, 032.

Hui, L. I. (2008). The analysis of factors which affect students' aerobics teaching ability which take aerobics as his specialty in PE department. Journal of Qiongzhou University, 5, 029.

Jin, J. L., Wei, Y. M., \& Jing, D. (2004). Fuzzy comprehensive evaluation model based on improved analytic hierarchy process. Journal of Hydraulic Engineering, 2004(2), 144-147.

Liu, Z. M. (2004). Discussion on the Method of Fostering Students' Ability to Teach Basic Gymnastics in PE Department. Journal of Physical Education Institute of Shanxi Teachers University.

Saaty, T. L. (2008). Decision making with the analytic hierarchy process. International journal of services sciences, 1(1), 83-98. http://dx.doi.org/10.1504/IJSSCI.2008.017590

Saaty, T. L. (1994). How to make a decision: The analytic hierarchy process. Interfaces, 24(6), 19-43. http://dx.doi.org/10.1287/inte.24.6.19

Sun, Z. G. (2004). Research on the indispensable teaching ability in physical education of the new century. Journal of Changchun Teachers College.

Zhao, L. P., Ling, M., Zhang, R. R., Hu, T. Y., Lin, Y. Q., \& Zhao, T. G. (2010). Cultivation of students' ability in provincial track and field courses. Journal of Yibin University, 6, 043.

Zou, X. L. (2014). Training of physical education students' gymnastics teaching ability under the new curriculum standard. Bulletin of Sport Science \& Technology. 